\title{
Erratum to: Fabrication of graphite-graphene-ionic liquid modified carbon nanotubes filled natural rubber thin films for microwave and energy storage applications
}

\author{
Srinivasarao Yaragalla ${ }^{1}$ - Bashaiah Sindam ${ }^{2}$. Jiji Abraham ${ }^{1}$ - K. C. James Raju ${ }^{2}$. \\ Nandakumar Kalarikkal ${ }^{1,3} \cdot$ Sabu Thomas ${ }^{1,4}$
}

Published online: 16 August 2015

(C) Springer Science+Business Media Dordrecht 2015

Erratum to: J Polym Res (2015) 22:137

DOI 10.1007/s10965-015-0776-5

Table 1 in the original publication contains a mistake. In the last row of the first column, "4" should have been " 5 ". The correct Table 1 is presented here.
Table 1 Formulations of NR composites (Amounts are taken in \% W/ W ratio)

\begin{tabular}{llllllll}
\hline TRG & NR & ZnO & Stearic acid & TMTM & TQ & CBS & Sulphur \\
\hline 0 & 100 & 5 & 2 & 0.1 & 3 & 1.5 & 2.5 \\
0.5 & 100 & 5 & 2 & 0.1 & 3 & 1.5 & 2.5 \\
1 & 100 & 5 & 2 & 0.1 & 3 & 1.5 & 2.5 \\
2 & 100 & 5 & 2 & 0.1 & 3 & 1.5 & 2.5 \\
3 & 100 & 5 & 2 & 0.1 & 3 & 1.5 & 2.5 \\
5 & 100 & 5 & 2 & 0.1 & 3 & 1.5 & 2.5 \\
\hline
\end{tabular}

The online version of the original article can be found at http://dx.doi.org/ 10.1007/s10965-015-0776-5.

K. C. James Raju

kcjrsp@uohyd.ernet.in

$\triangle$ Nandakumar Kalarikkal

nkkalarikkal@mgu.ac.in

Sabu Thomas

sabupolymer@yahoo.com

1 International and Inter University Centre for Nanoscience and Nanotechnology, Mahatma Gandhi University,

Kottayam 686560, Kerala, India

2 School of Physics, University of Hyderabad,

Hyderabad 500046, Telengana, India

3 School of Pure and Applied Physics, Mahatma Gandhi University,

Kottayam 686560, Kerala, India

4 School of Chemical Sciences, Mahatma Gandhi University,

Kottayam 686560, Kerala, India 